\title{
Congenital Langerhans cell histiocytosis: a good prognosis disease?*
}

\author{
Ana Paula Frade ${ }^{1}$ \\ Anna Beatriz Willemes Batalha ${ }^{3}$
}

\author{
Mariana Marteleto Godinho² \\ Ana Paula Silva Bueno ${ }^{3}$
}

DOI: http:/ / dx.doi.org/10.1590/abd1806-4841.20175308

\begin{abstract}
Langerhans cell histiocytosis is rare and more frequent in children. The skin is affected in $50 \%$ of the cases and is the only site in $10 \%$. Its course varies from self-limited and localized forms to severe multisystemic forms. Congenital cases are usually exclusively cutaneous and self-limited, with spontaneous remission in months. This study presents a rare congenital case, initially restricted to the skin, with subsequent dissemination and fatal outcome. A male newborn presented congenital disseminated erythematous scaly lesions. The biopsy was conclusive for Langerhans cell histiocytosis. The patient evolved into the multisystemic form in weeks, when chemotherapy was started, according to the LCH-2009 protocol; however, the patient was refractory to treatment and died.
\end{abstract}

Keywords: Congenital abnormalities; Child; Histiocytosis; Histiocytosis, Langerhans-cell; Infant, newborn, diseases

\section{INTRODUCTION}

Langerhans cell histiocytosis $(\mathrm{LCH})$ is rare and results from clonal proliferation of cells, which are similar to Langerhans cells (LC). Its incidence is three to five cases per million. It may appear at any age, but it is most frequent in children. ${ }^{1,2}$ In the neonatal period, incidence is one to two cases per million. ${ }^{3}$

The most affected organs are bones and lymph nodes. The skin is affected in $50 \%$ of the cases and is the only site in $10 \%$. Its course varies from self-limited and localized forms to severe multisystemic forms. The study presents congenital case initially restricted to the skin, with dissemination and fatal outcome.

\section{CASE REPORT}

A male full-term newborn, in good health conditions, upon birth presented erythematous plaques and papules, covered with disseminated brownish crusts and erythematous macerated lesions in the inguinal region (Figures 1 to 4). At four days old, our medical team decided to perform an incisional biopsy of the trunk lesion.
Serologies for congenital infectious diseases, blood count, biochemistry, and maternal autoantibodies (FAN, anti-Ro, anti-La) presented normal results. Histopathological examination showed cells with lobed core permeating the epidermis. The immunohistochemical study was positive for Cd1a in mononuclear cells from the high dermis and S100 in the epidermis, reaching a diagnosis of LCH (Figure 5).

Laboratory screening for systemic disease through hematology, according to the LCH-2009 Protocol from the Histiocytosis International Society, showed mild anemia (Hct 29\%, Hgb 10.0g/dl) and hypoalbuminemia $(2.4 \mathrm{~g} / \mathrm{dl})$. Normal urine osmolarity, skeletal $\mathrm{X}$-ray, and upper GI endoscopy were performed. Abdominal ultrasound showed the liver in the upper limit of normality and a slightly enlarged spleen. The bone marrow biopsy did not obtain enough material for analysis, and the liver biopsy showed nonspecific inflammatory changes.

In only a few weeks, the patient developed hepatosplenomegaly, hypoalbuminemia, hypofibrinogenemia, worsening anemia,

\section{Work submitted on 29.10.2015}

Approved by the Advisory Board and accepted for publication on 01.05.2016

* Work performed at the Instituto de Pediatria Martagão Gesteira, Universidade Federal do Rio de Janeiro (IPPMG/UFRJ), Rio de Janeiro (RJ), Brazil. Financial support: None

Conflict of interest: None

Secretaria Municipal de Saúde do Rio de Janeiro, Rio de Janeiro (RJ), Brazil.

Private Clinic, Rio de Janeiro (RJ), Brazil.

Department of Hematology, Instituto de Pediatria Martagão Gesteira, Universidade Federal do Rio de Janeiro (IPPMG-UFRJ) - Rio de Janeiro (RJ), Brazil.

()2017 by Anais Brasileiros de Dermatologia 
and thrombocytopenia. Chest computed tomography showed subpleural bubbles. These findings have defined the progression to the systemic form and chemotherapy was begun with prednisone and vinblastine, according to that set forth in the LCH-2009 protocol.

In the fifth week of treatment, the patient developed suppurative otorrhea in the right ear without general symptoms. It was difficult to establish whether there was progression of disease with extension to another organ (bone), whether the clinical picture was secondary to otitis externa due to the disease itself or whether the picture was of infectious nature. The infant was hospitalized and treated with cefepima, producing a good response and complete resolution of the lesion after seven days of treatment. The final diagnosis was necrotizing otitis externa.

In the sixth week the patient was classified as "stable disease with involvement of target organ", and the protocol was followed as planned, with intravenous chemotherapy for six more weeks.

In the 12th week reassessment, there were signs of progression of the disease, with a worsening of the skin lesions and hema-

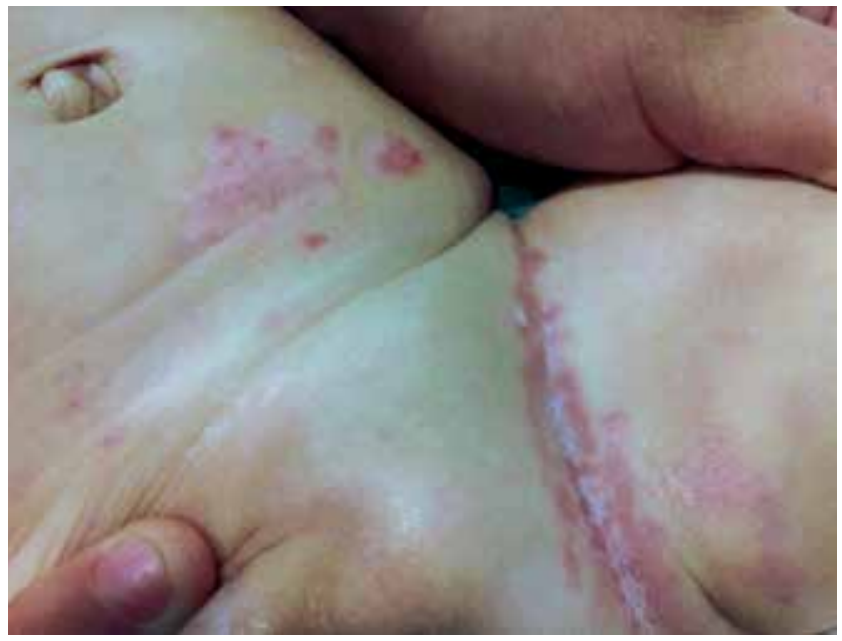

Figure 1: Erythematous plaques in the inguinal area

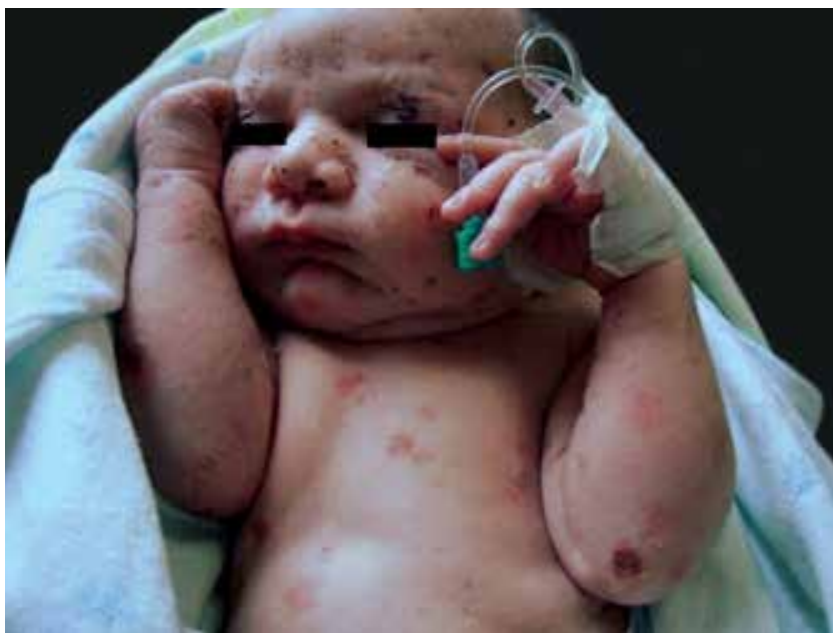

FIGURE 2: Disseminated erythematous plaques and papules, covered with brownish crusts tological parameters. The examination showed ulcerated lesions in the lower abdomen and inguinal region, crusted lesions in the pinna and retroauricular regions. According to the $\mathrm{LCH}-2009$ protocol, the patient was hospitalized and underwent salvage chemotherapy (cladribine + cytarabine). The patient presented infectious complications and severe pancytopenia, leading to the patient's admission to the ICU. The patient also continued to develop hepatosplenomegaly and recurrent otitis. In view of the disease progression, the patient received chemotherapy for refractory multisystemic disease, and died 11 months later due to septic shock.

\section{DISCUSSION}

Skin lesions are present in 50\% of the cases. Manifestations are varied, but characteristically affect the scalp and flexures. Violet nodules, erythematous plaques, and papules covered with hematic brownish crusts, vesicles, exulcerations, and tumors were observed. ${ }^{4}$

Four well-defined clinical forms have been described: Letterer-Siwe or acute disseminated disease, Hand-Schüller-Christian

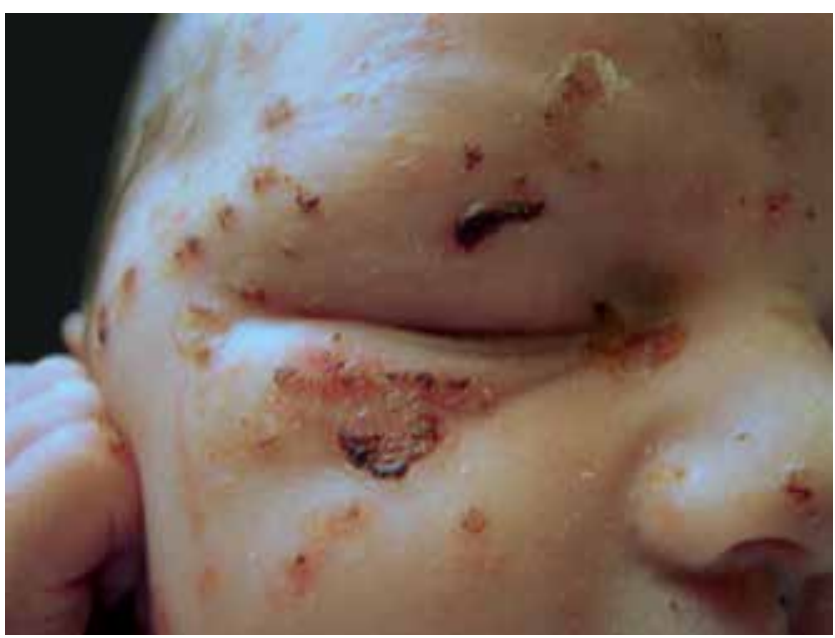

Figure 3: Erythematous plaques with hematic crusts

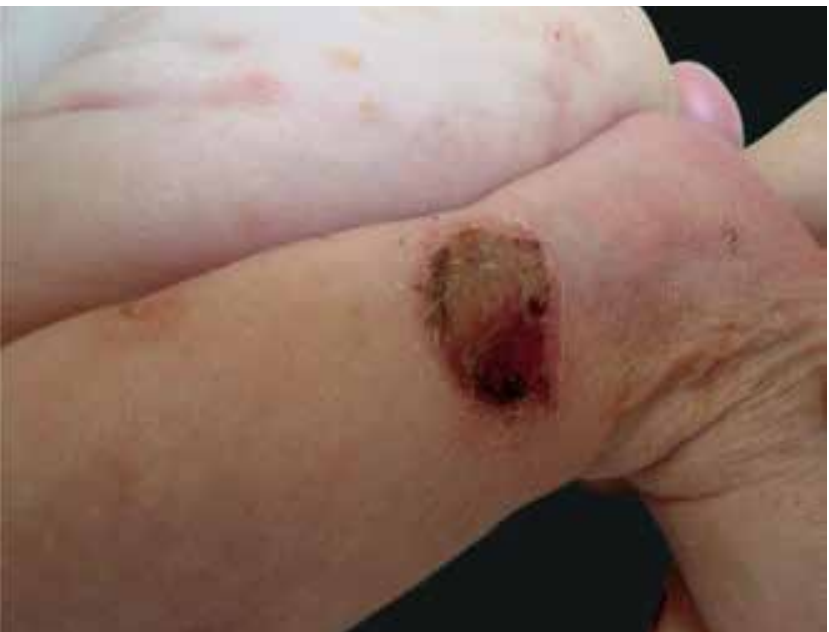

Figura 4: Exulceration covered with brownish crusts 


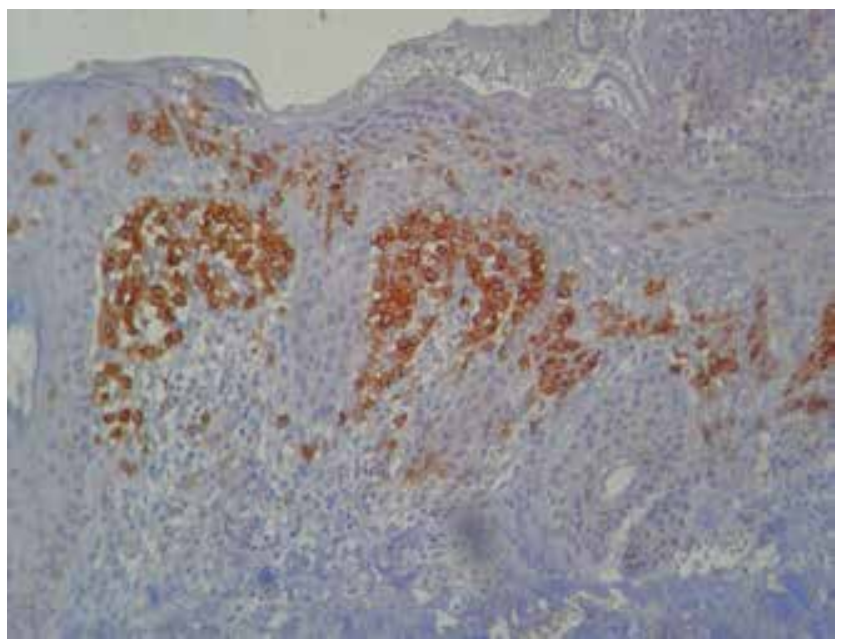

Figure 5: Immunohistochemical study showing positive Cd1a in mononuclear cells from the high dermis. IHQ, X40

or multifocal chronic disease, focal or eosinophilic granuloma, and Hashimoto Pritzker disease. ${ }^{5}$ The congenital cases described in these studies are mostly forms that are exclusively cutaneous and self-limited, with spontaneous remission occurring in months. There are rare cases reported of the progression of the diseases, together with the compromising of other organs. Even more rare are studies with fatal outcomes. ${ }^{6}$

Extensive skin lesions do not indicate severity, and all patients must be followed up even after spontaneous remission. ${ }^{3}$ There are no reliable clinical and laboratory predictors that identify patients at risk of developing the multisystem form. ${ }^{7}$

\section{REFERENCES}

1. Minkov M. Multisystem Langerhans cell histiocytosis in children: current treatment and future directions. Paediatr Drugs. 2011;13:75-86

2. Margo CE, Goldman DR. Langerhans Cell Histiocytosis. Surv Ophthalmol. 2008;53:332-58.

3. Stein SL, Paller AS, Haut PR, Mancini AJ. Langerhans cell histiocytosis presenting in the neonatal period: A retrospective case series. Arch Pediatr Adolesc Med. 2001:155:778-83

4. Aricò M, Egeler RM. Clinical aspects of Langerhans cell histiocytosis. Hematol Oncol Clin North Am. 1998;12:247-58

5. Goodman W, Barret T. Histiocytoses. Philadelfia: Mosby; 2003

6. Fuentes Castelló MA, Montahud Posada C, Belenguer Font MJ, Pérez Ramos M, Vargas Torcal F. Congenital presentation of nonself-healing Langerhans' cell histiocytosis. An Pediatr (Barc). 2006;65:634-5
In 2009, the Histiocyte Society classified LCH into two groups: single organ disease and multisystemic disease. ${ }^{8}$ Such a classification highlights the need to screen lesions in other organs for all the patients with a confirmed diagnosis, regardless of their clinical picture, together with guided treatment.

The diagnosis should be considered in cases of seborrheic dermatitis and diaper dermatitis which have not presented good response to treatment. The purpuric aspect of the lesions should take such diagnosis into consideration. Histopathological examination and immunohistochemical analysis are diagnostic. The main criterion is positivity for CD1a and/or Langerin (CD207).

The treatment of the multisystemic form consists of a first cycle of chemotherapy with vinblastine and prednisone for six weeks. A second cycle is recommended for patients with at-risk or affected organs (liver, spleen, bone marrow) who demonstrated partial or total response, as well as for those who presented no involvement of at-risk organs initially, but showed no remission with treatment. Second line therapy is indicated when there are persistent lesions in at-risk organs after the second cycle of chemotherapy or disease progression within six weeks. There is no definition of the best treatment for patients who are refractory to initial therapy. The prognosis of these patients is reserved, with a three-year survival of $17 \%$. Promising results were also obtained with the use of cytarabine. ${ }^{9}$

Currently, LCH is understood as a spectral disease with a wide variation of clinical presentations and unpredictable clinical course. LCH is rarely congenital. This study presents a case of congenital LCH with only skin lesions initially, followed by dissemination to other organs, refractory reaction to treatment, and a fatal outcome. It is important to recognize the need to monitor patients over the long term, regardless of the clinical form of presentation concerning the risk of dissemination, as well as to achieve an early histological diagnosis.]
7. Kapur P, Erickson C, Rakheja D, Carder KR, Hoang MP. Congenital self-healing reticulohistiocytosis (Hashimoto-Pritzker disease): Ten-year experience at Dallas Children's Medical Center. J Am Acad Dermatol. 2007;56:290-4

8. Histiocyte Society. Langerhans Cell Histiocytosis: Evaluation and Treatment Guidelines. April 2009.

9. Abla 0, Egeler RM, Weitzman S. Langerhans cell histiocytosis: Current concepts and treatments. Cancer Treat Rev. 2010;36:354-9.

\section{MAILING ADDRESS: \\ Ana Paula Frade Lima Pinto \\ Avenida Bruno Lobo, 50 \\ Cidade Universitária \\ 21941-590 Ilha do Fundão, RJ \\ Brazil \\ E-mail: anapaulafrade@hotmail.com}

How to cite this article: Frade AP, Godinho MM, Batalha ABW, Bueno APS. Congenital Langerhans cell histiocytosis: a good prognosis disease? An Bras Dermatol. 2017;92(5 Suppl 1): 40-2. 